\title{
4 BULlying NO TRABALHO: PERCEÇÃO E IMPACTO NA SAÚdE MENTAL E VIDA PESSOAL DOS ENFERMEIROS
}

| Antónia Teixeira'; Teresa Ferreira²; Elisabete Borges ${ }^{3} \mid$

\section{RESUMO}

CONTEXTO: O bullying é um tipo de violência que tem vindo a ser analisado em vários contextos. O setor da saúde é um dos locais onde tem sido descrita a sua incidência, constituindo uma ameaça à saúde mental dos profissionais, podendo colocar em causa a segurança dos cuidados prestados aos cidadãos.

OBJETIVOS: Descrever as perceções dos enfermeiros sobre bullying no trabalho e o seu impacto na saúde mental e vida pessoal destes profissionais.

METODOLOGIA: O estudo foi dirigido a enfermeiros com 10 ou mais anos de atividade profissional. Através da técnica de amostragem por redes foram selecionados 12 participantes, a quem foram realizadas entrevistas semiestruturadas.

RESULTADOS: Da perceção do conceito de bullying no trabalho, salienta-se que a maioria dos enfermeiros não refere duas das principais dimensões deste fenómeno, a frequência e a duração dos comportamentos agressivos. Stresse, ansiedade, tristeza, irritabilidade, intenção de mudar de serviço, cansaço, insónias, perturbações digestivas e isolamento são algumas consequências na saúde mental deste fenómeno referidas pelas vítimas.

CONCLUSÕES: O bullying é um fenómeno que tem consequências negativas para a saúde mental e vida dos enfermeiros. A formação sobre bullying no trabalho e a capacitação dos enfermeiros para gerir o stresse resultante são propostas para minimização das consequências deletérias do bullying na saúde mental destes profissionais, de forma a promover a sua saúde em geral.

PALAVRAS-CHAVE: Bullying; Saúde mental; Saúde ocupacional; Enfermeiros

\section{RESUMEN}

"Acoso laboral: Percepción e impacto en la salud mental y en la vida personal de los enfermeros"

CONTEXTO: El acoso es un tipo de violencia que se ha analizado en diversos contextos. El sector sanitario es uno de los contextos donde se ha descrito su incidencia, ya que constituye una amenaza para la salud mental de los profesionales, que puede poner en entredicho la seguridad de los cuidados prestados a los ciudadanos.

OBJETIVOS: Describir las percepciones de los enfermeros sobre el acoso en el trabajo y su impacto en la salud mental y en la vida personal de estos profesionales.

METODOLOGÍA: El estudio se ha dirigido a enfermeros con $10 \mathrm{o}$ más años de actividad profesional. A través de la técnica de muestreo por redes, se han seleccionado 12 participantes, a quienes se han realizado entrevistas semiestructuradas.

RESULTADOS: De la percepción del concepto de acoso en el trabajo, cabe destacar que la mayoría de los enfermeros no señala dos de las principales dimensiones de este fenómeno: la frecuencia y la duración de los comportamientos agresivos. Estrés, ansiedad, tristeza, irritabilidad, intención de cambiar de servicio, cansancio, insomnio, perturbaciones digestivas y aislamiento son algunas de las consecuencias de este fenómeno en la salud mental que apuntan las víctimas.

CONCLUSIONES: El acoso es un fenómeno que tiene consecuencias negativas para la salud mental y la vida de los enfermeros. Se propone la formación sobre acoso en el trabajo y la capacitación de los enfermeros para gestionar el estrés resultante y de esa forma minimizar las consecuencias nocivas del acoso en la salud mental de estos profesionales, a fin de fomentar su salud en general.

\section{DESCRIPTORES: Violencia laboral; Salud mental; Salud lab-} oral; Enfermeros

\begin{abstract}
"Bullying at Work: Perception and impact on the mental health and personal life of nurses"

BACKGROUND: Bullying is a form of violence that has been analysed in various contexts. The health sector is one of the contexts where its incidence has been described which poses a threat to the professionals' mental health that may undermine the safety of care provided to citizens.

AIMS: To describe the nurses' perceptions about bullying at work and its impact on the mental health and personal lives of these professionals. METHODOLOGY: The study was directed to nurses with 10 or more years of professional activity. Through the technique of network sampling, we selected 12 participants, to whom semi-structured interviews were conducted.

RESULTS: From the perception of the concept of bullying at work, it is noted that most nurses do not regard neither the two major dimensions of this phenomenon nor the frequency and duration of the aggressive behaviour. Stress, anxiety, sadness, irritability, intention to change service, fatigue, insomnia, digestive disorders and isolation are some mental health consequences of this phenomenon referred by the victims.

CONCLUSIONS: Bullying is a phenomenon which has negative consequences for the mental health and life of nurses. Training in bullying at work and in nurses' ability to manage the resulting stress are proposed with the aim of minimising the deleterious consequences of bullying in these professionals' mental condition, in order to promote their overall health.
\end{abstract}

\section{KEYWORDS: Bullying; Mental health; Occupational health; Nurses}

Submetido em 30-09-2015

Aceite em 30-03-2016

1 Mestre em Enfermagem Comunitária; Enfermeira no Centro Hospitalar São João, E.P.E., Alameda Prof. Hernâni Monteiro, Porto, Portugal, u009637@hsjoao.min-saude.pt 2 Doutora em Psicologia da Saúde; Professora Coordenadora na Escola Superior de Enfermagem do Porto, Porto, Portugal, teresarodrigues@esenf.pt

3 Doutora em Enfermagem; Professora Adjunta na Escola Superior de Enfermagem do Porto, Rua Dr. António Bernardino de Almeida, Porto, Portugal, elizabete@esenf.pt

Citação: Teixeira, A., Ferreira, T. \& Borges, E. (2016). Bullying no trabalho: Perceção e impacto na saúde mental e vida pessoal dos enfermeiros. Revista Portuguesa de Enfermagem de Saúde Mental (15), 23-29. 


\section{INTRODUÇÃO}

O trabalho faz parte da natureza humana, sendo essencial que se desenvolva num ambiente seguro e saudável, de forma a preservar a saúde mental e promover a saúde em geral do ser humano. São vários os fatores que ameaçam a segurança e saúde no local de trabalho, entre eles a violência. Quando os comportamentos agressivos se prolongam no tempo, de forma repetida e com intencionalidade, estamos perante o fenómeno denominado por bullying (Einarsen, Hoel, Zapf \& Cooper, 2011).

Os autores que têm estudado esta problemática utilizam termos diversos, tais como: assédio moral (Hirigoyen, 2002; Sá, 2008); mobbing (Carvalho, 2007; Leymann, 1996; Saraiva e Pinto, 2011) e bullying (Araújo, 2009; Borges e Ferreira, 2015; Einarsen et al., 2011; Simons \& Mawn, 2010; Townsend, 2012). O conceito de violência no trabalho é extenso e complexo, daí as inúmeras definições, mas, apesar de algumas particularidades entre as diferentes terminologias para descrever o fenómeno, elas salientam o facto de o comportamento ser ofensivo, repetido e continuado no tempo, contra uma ou mais pessoas (European Agency for Safety and Health at Work [EU-OSHA], 2007).

Apesar das diferentes terminologias, neste trabalho opta-se pela utilização do termo bullying, uma vez que é o termo mais utilizado na bibliografia consultada. No entanto, ao longo do texto utilizaremos o termo que o autor em referência utiliza, preservando a denominação original.

São muitas as causas possíveis para este fenómeno (Zapf \& Einarsen, 2011a), que pode ser causado por mais do que um fator simultaneamente (Zapf ,1999). A liderança, a cultura organizacional, fatores stressantes do trabalho e a organização do trabalho, estão entre as causas organizacionais (Zapf, 1999). Em Portugal, Araújo (2009) constatou que uma maior satisfação com o clima organizacional contribui para menores índices de bullying. Num estudo com enfermeiros, Sá (2008) averiguou que a maioria das situações de assédio ocorreu após uma mudança organizacional. Também num estudo numa população de enfermeiros, Saraiva e Pinto (2011) concluíram que a satisfação profissional influencia os índices de mobbing, verificando que as pessoas insatisfeitas com a profissão são as que sofrem mais condutas de mobbing.

Outros fatores podem ser causadores de bullying, como a ambição de poder (Strandmark \& Hallberg, 2007); fatores sociais, como hostilidade, inveja e pressão de grupo, e fatores pessoais do agressor e da vítima (Zapf, 1999).

Leymann (1996) refere que o stresse pode ser tanto uma causa como uma consequência de mobbing, em que determinadas situações, como más condições psicossociais no local de trabalho, podem originar reações de stresse, que podem tornar-se, por sua vez, fatores de stresse para outras pessoas, promovendo ações de mobbing. Também Zapf (1999) alude a esta relação bidirecional entre causas e consequências, referindo que consequências de mobbing, como ansiedade, comportamento depressivo e obsessivo, podem produzir reações negativas no grupo que, após algum tempo, podem levar a comportamentos de mobbing. Existe a possibilidade de um ciclo vicioso, em que o bullying no trabalho e as consequências a nível da saúde mental potenciam-se reciprocamente de forma negativa (Reknes et. al, 2014).

O tempo é um importante critério para diferenciar entre bullying e os conflitos quotidianos nas organizações e, ainda que a utilização de uma duração mínima dependa do contexto de trabalho, mesmo sem um prazo concreto, há consenso entre os investigadores que o bullying é uma questão de meses e anos e não de dias e semanas (Einarsen et al., 2011).

Está presente em vários contextos laborais, entre eles, o dos enfermeiros (Borges e Ferreira, 2015). Devido às suas características, a literatura tem demonstrado que este tipo de violência é responsável por graves consequências na saúde física e mental das pessoas envolvidas, conduzindo também a consequências para as organizações e para toda a sociedade em geral. Estas consequências, nefastas, afetam o desempenho dos profissionais, colocando em causa a qualidade dos cuidados prestados à população (Townsend, 2012), uma vez que contribui para erros, que incidem negativamente nas pessoas recetoras dos cuidados de enfermagem (Mitchell, Ahmed, \& Szabo, 2014).

Este trabalho, cujo objetivo é descrever as perceções dos enfermeiros sobre bullying no trabalho e o impacto das experiências de bullying na sua saúde mental e vida pessoal, tem como finalidade sensibilizar os enfermeiros para este fenómeno, de forma a contribuir para a preservação da saúde mental destes profissionais em contexto laboral.

O presente estudo é do tipo exploratório, descritivo e transversal e insere-se numa perspetiva qualitativa, de orientação fenomenológica. 


\section{METODOLOGIA}

A importância do bullying no local de trabalho para a Enfermagem, com as suas implicações quer para a saúde dos profissionais, quer para a qualidade dos cuidados por estes prestados, serviu de ponto de partida para este estudo. A sua realização teve como objetivos: descrever as perceções dos enfermeiros sobre bullying no trabalho e descrever o impacto das experiências de bullying na saúde mental e vida pessoal destes profissionais.

Este estudo é do tipo exploratório, descritivo e transversal, inserido no paradigma de investigação qualitativa. Foi dirigido a enfermeiros com, no mínimo, 10 anos de exercício profissional. O método de seleção dos participantes foi a amostragem por redes. $\mathrm{O}$ número de participantes foi condicionado pela saturação da informação, sendo no total 12 enfermeiros. A entrevista semiestruturada foi a técnica utilizada para a recolha de informação, que decorreu no período compreendido entre 16 de Abril e 30 de Maio de 2012. A informação fornecida durante a entrevista foi áudio gravada, posteriormente transcrita e submetida a uma análise de conteúdo categorial de Bardin (2009). Foram cumpridos os requisitos éticos do processo investigativo.

Quanto ao grupo de participantes, este é predominantemente do sexo feminino (83,3\%). A média de idades é de 35,9 anos $(\mathrm{DP}=4,8)$, correspondendo a idade mínima a 31 anos e a idade máxima a 49 anos. Relativamente ao estado civil, $50 \%$ dos participantes são solteiros e os restantes $50 \%$ são casados. Quanto à formação profissional, dos 12 participantes, sete $(58,3 \%)$ possuem o Curso de Licenciatura em Enfermagem e cinco (41,7\%) possuem formação pós-graduada. Todos os participantes têm a categoria de enfermeiro. $\mathrm{O}$ tempo médio de experiência profissional é de 13,7 anos $(\mathrm{DP}=4,3)$. Apenas um dos participantes $(8,3 \%)$ exerce funções no serviço atual há menos de um ano. A maioria dos participantes $(83,3 \%)$ trabalha em contexto hospitalar.

\section{RESULTADOS}

A identificação de casos de bullying foi necessária para se proceder à descrição das vivências dos participantes relativamente a este fenómeno.

Dos 12 participantes, seis consideram que já foram vítimas de bullying no trabalho. No entanto, como a descrição efetuada por dois destes participantes se referiu a casos pontuais, consideraram-se atos de bullying os descritos por quatro participantes.

Nenhum dos participantes se reconheceu como agressor.
Procedemos, de seguida, à discussão das categorias: conceito de bullying no trabalho e impacto das experiências de bullying na saúde mental e vida pessoal dos enfermeiros.

\section{DISCUSSÃO}

\section{Conceito de Bullying no Trabalho}

Quando questionados sobre o que entendiam por bullying no trabalho, os participantes referiram-se ao âmbito, aos intervenientes, à temporalidade e consequências do fenómeno, como se mostra na tabela 1 .

Tabela 1 - Conceito de Bullying no Trabalho

\begin{tabular}{|c|c|c|}
\hline \multicolumn{3}{|c|}{ CATEGORIA - CONCEITO DE BULLYING NO TRABALHO } \\
\hline $\begin{array}{l}\text { Subcatego- } \\
\text { ria }\end{array}$ & Unidades de Registo & $\begin{array}{l}\text { Unidades } \\
\text { de Enume- } \\
\text { ração }\end{array}$ \\
\hline $\begin{array}{l}\text { Tipo Psi- } \\
\text { cológico }\end{array}$ & $\begin{array}{l}\text { “... chantagem psicológica...” (E1) } \\
\text { “... violência psicológica...” (E2) } \\
\text { “... pressão psicológica...” (E3) } \\
\text { “... às vezes verbal...” (E4) } \\
\text { “... mais verbal, psicológico.... ser agredida ver- } \\
\text { balmente ou mesmo usarem palavrões ou falarem } \\
\text { alto...” (E5) } \\
\text { “... perseguição... pressionar psicologicamente, } \\
\text { emocionalmente...” (E6) } \\
\text { “... é quando uma pessoa... não tem um ambiente } \\
\text { de trabalho... facilitador... sujeita a situações } \\
\text { menos agradáveis...” (E7) } \\
\text { “... sofrer algum tipo de represálias... sentir-se } \\
\text { ameaçada...” (E8) } \\
\text { “... algum tipo de agressão, pressão, no trab- } \\
\text { alho...” (E10) } \\
\text { “... agressão verbal... irritação...” (E11) } \\
\text { “... passa mais por descriminação... violência } \\
\text { psicológica...” (E12) }\end{array}$ & 11 \\
\hline Tipo Físico & $\begin{array}{l}\text { “... física é muito raro acontecer...” (E1) } \\
\text { “... violência... física, no local de trabalho...” (E2) } \\
\text { “... pode ser físico...” (E4) }\end{array}$ & 3 \\
\hline $\begin{array}{l}\text { Tipo Físico e } \\
\text { psicológico }\end{array}$ & $\begin{array}{l}\text { “... uma certa forma de agressão, que pode ser } \\
\text { direta ou indireta..." (E9) }\end{array}$ & 1 \\
\hline Tipo Sexual & “... pode haver algum assédio sexual..." (E12) & 1 \\
\hline $\begin{array}{l}\text { Intervenien- } \\
\text { te - Agressor }\end{array}$ & $\begin{array}{l}\text { “... colegas...” (E1, E2, E10, E11) } \\
\text { “... pelos pares... pelas outras categorias profis- } \\
\text { sionais... pelo doente... pelos familiares..." (E3) } \\
\text { “... pelos familiares..." (E5) } \\
\text { “... tanto da parte de colegas... visitas... e doen- } \\
\text { tes..." (E6) } \\
\text { “...porpartedosoutros elementos da equipa...”(E7) }\end{array}$ & 8 \\
\hline $\begin{array}{l}\text { Intervenien- } \\
\text { te - Vítima }\end{array}$ & “... personalidade mais frágil...” (E1) & 1 \\
\hline $\begin{array}{l}\text { Intencionali- } \\
\text { dade }\end{array}$ & $\begin{array}{l}\text { “... de propósito...” (E8) } \\
\text { “... no sentido de... os tentarem prejudicar...” } \\
\text { (E12) }\end{array}$ & 2 \\
\hline $\begin{array}{l}\text { Temporali- } \\
\text { dade }\end{array}$ & $\begin{array}{l}\text { “... eu penso que é uma coisa continuada no } \\
\text { tempo...” (E2) } \\
\text { “... a que nós somos sujeitos no dia-a-dia...” (E3) }\end{array}$ & 2 \\
\hline $\begin{array}{l}\text { Consequên- } \\
\text { cias }\end{array}$ & $\begin{array}{l}\text { “... a pessoa sente-se um bocadinho à margem...” } \\
\text { (E7) } \\
\text { “... fazer outra pessoa passar bastante mal..." (E8) } \\
\text { “... vai influenciar a atividade... a forma de estar } \\
\text { da pessoa...” (E9) } \\
\text { “... fazerem-te sentir mal, quer enquanto profis- } \\
\text { sional, quer enquanto pessoa...” (E11) }\end{array}$ & 4 \\
\hline
\end{tabular}


Quase todos os participantes concetualizam o bullying no âmbito psicológico. O bullying no trabalho é considerado como chantagem, pressão, perseguição, agressão verbal, ameaça e descriminação. No entanto, a maioria dos participantes apenas faz referência a uma destas características do bullying.

A natureza física do bullying também é referenciada por alguns participantes, mas evidenciam que é menos frequente que a natureza psicológica do fenómeno. $\mathrm{O}$ bullying engloba diferentes tipos de comportamentos, tais como insultos ofensivos persistentes, crítica persistente e abuso pessoal ou mesmo abuso físico (Zapf, Escartín, Einarsen, Hoel, \& Vartia, 2011b), mas a sua natureza é essencialmente psicológica (Einarsen et al., 2011).

Quase todos os participantes referenciam os colegas como os principais agressores, sendo também referenciados os elementos de outras classes profissionais, os familiares dos doentes e os doentes. Na sua investigação, Carvalho (2007) constatou que o assédio do tipo horizontal foi o mais referido, em que os enfermeiros foram assediados, na sua maioria, por um ou vários colegas. Quanto à intencionalidade, dois participantes referem que existe intenção por parte dos agressores. Segundo Hirigoyen (2002), no assédio moral há uma intenção, consciente ou não, de prejudicar o outro e as consequências tornam-se mais graves quando a pessoa se apercebe de que existe intenção de a magoar. Apesar de ser difícil verificar se existe intenção, esta não altera a situação das vítimas, e os comportamentos ofensivos devem ser considerados, independentemente da intenção (Einarsen et al., 2011).

Apenas dois participantes se referiram à temporalidade, percecionando o bullying como algo que se prolonga no tempo. A temporalidade é uma das dimensões do conceito de bullying. Zapf et al. (2011b) mencionam que a duração do bullying é um importante critério para diferenciar entre bullying e os conflitos quotidianos nas organizações. A frequência e duração dos comportamentos negativos indesejados parecem ser tão importantes quanto a natureza dos mesmos (Einarsen et al., 2011).

Relativamente às consequências, os participantes referem que este tipo de violência influencia as pessoas e a própria atividade, sendo responsável por sentimentos negativos e de marginalização. As consequências também estão inerentes na definição proposta por Einarsen et al. (2011), onde bullying significa assediar, perseguir, ofender ou excluir socialmente ou afetar negativamente o seu trabalho.
Outra dimensão presente na definição de bullying referida por Einarsen et al. (2011) é a desigualdade de poder entre as partes envolvidas, em que o bullying é descrito como um processo crescente, em escalada, e que a pessoa confrontada acaba numa posição inferior, passando a ser alvo de comportamentos de bullying. Também Hirigoyen (2002) refere que o assédio moral é precedido por uma influência psicológica do agressor e de uma submissão forçada da vítima, considerando, assim, uma parte dominadora e outra dominada. Da informação obtida não se encontraram dados que se referissem a esta dimensão.

De referir que a noção de bullying em ambiente escolar foi evidenciada por dois participantes, como ilustram as seguintes unidades de registo: “... a minha noção mais de bullying é até nas crianças, na escola...” (E8); “... é mais usado nas escolas, é por isso que tem mais impacto..." (E10). Fica manifesto que a perceção de bullying no trabalho apresenta limitações ao nível do conceito.

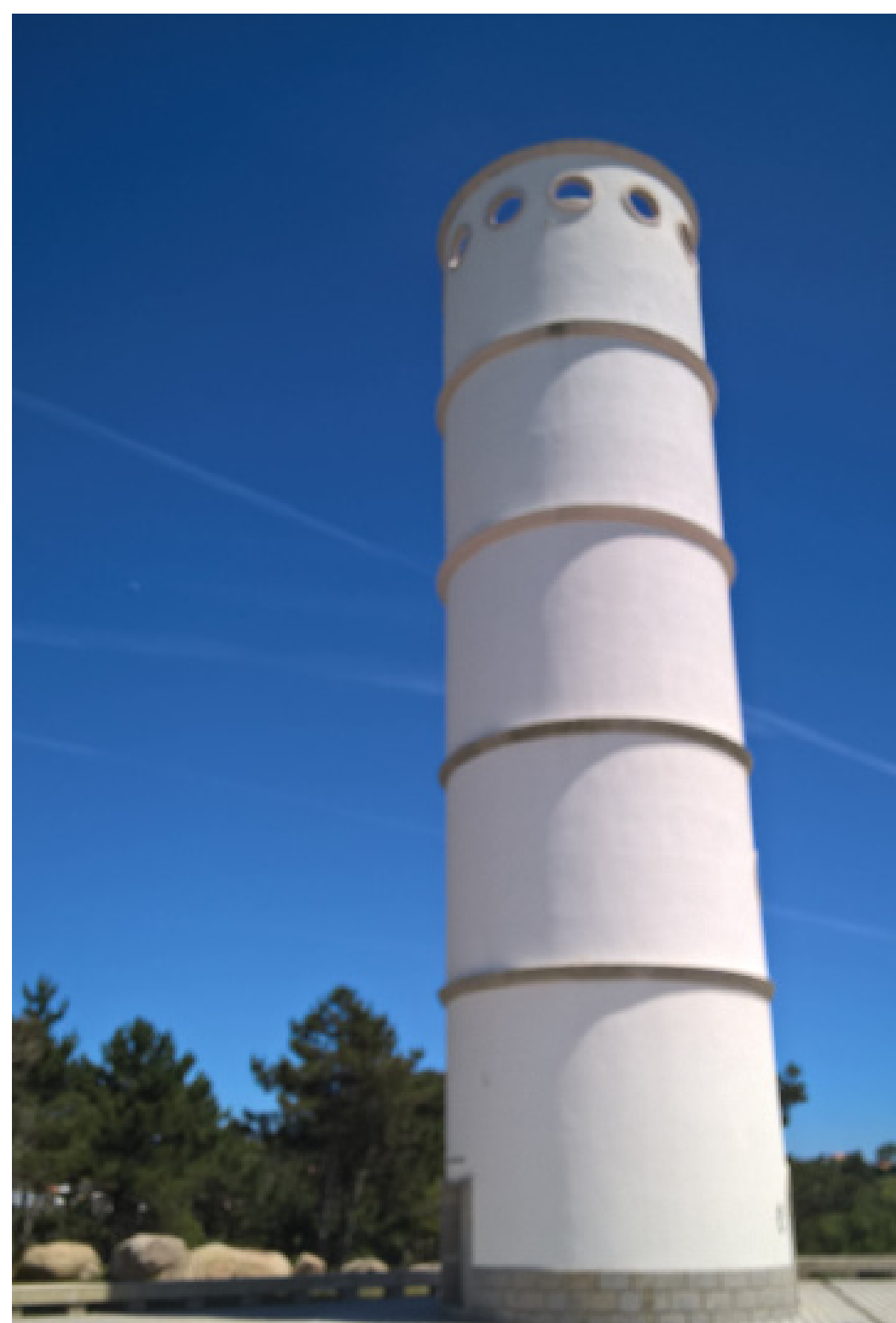




\section{Impacto das Experiências de Bullying na Saúde Mental e Vida Pessoal dos Enfermeiros}

As consequências dos atos de bullying vivenciados pelos participantes, enquanto vítimas, são descritas na tabela 2.

Tabela 2 - Impacto das Experiências de Bullying na Saúde Mental e Vida Pessoal dos Enfermeiros

\begin{tabular}{|c|c|c|}
\hline \multicolumn{3}{|c|}{$\begin{array}{l}\text { CATEGORIA - IMPACTO DAS EXPERIÊNCIAS DE BULLYING } \\
\text { NA SAÚDE MENTAL E VIDA PESSOAL DOS ENFERMEIROS }\end{array}$} \\
\hline $\begin{array}{l}\text { Subcatego- } \\
\text { ria }\end{array}$ & Unidades de Registo & $\begin{array}{l}\text { Unidades } \\
\text { de Enu- } \\
\text { meração }\end{array}$ \\
\hline Ansiedade & $\begin{array}{l}\text { “... A maior parte das vezes fico mais ansiosa...” (E1) } \\
\text { “... gerou um bocado de ansiedade...” (E7) } \\
\text { “... isso incomodava... já ia trabalhar com medo d'ir } \\
\text { trabalhar, de não saber o que me esperava naquele } \\
\text { turno... não só em termos de trabalho, mas também } \\
\text { em termos daquilo que as pessoas diziam...” (E11) }\end{array}$ & 3 \\
\hline Stresse & “... mais stressada...” (E1) & 1 \\
\hline Tristeza & $\begin{array}{l}\text { “... andava mais triste..." (E9) } \\
\text { “... acabava por chegar a casa e chorar... muita } \\
\text { tristeza... sempre mais deprimida..." (E11) }\end{array}$ & 2 \\
\hline $\begin{array}{l}\text { Irritabili- } \\
\text { dade }\end{array}$ & $\begin{array}{l}\text { “... mudanças de humor... acabo por ser intolerante } \\
\text { com pessoas que não têm nada a ver com o que } \\
\text { aconteceu..." (E1) }\end{array}$ & 1 \\
\hline $\begin{array}{l}\text { Intenção de } \\
\text { mudar de } \\
\text { serviço }\end{array}$ & $\begin{array}{l}\text { “.. a vontade que eu tive em determina- } \\
\text { da altura foi, até de mudar de serviço..." (E9) }\end{array}$ & 1 \\
\hline Ponderação & $\begin{array}{l}\text { “... questionar um bocado se era mesmo aquilo que } \\
\text { eu queria...” (E11) }\end{array}$ & 1 \\
\hline Cansaço & $\begin{array}{l}\text { “... mais cansada...” (E1) } \\
\text { “... cansaço extremo..." (E7) }\end{array}$ & 2 \\
\hline Insónias & “... insónias...” (E1) & 1 \\
\hline $\begin{array}{l}\text { Pertur- } \\
\text { bações } \\
\text { digestivas }\end{array}$ & “... a gastrite que volta e meia aparece...” (E1) & 1 \\
\hline Isolamento & $\begin{array}{l}\text { “... Senti-me num isolamento muito grande...” (E7) } \\
\text { “... Sentia pouca vontade de vir... apetecia-me era } \\
\text { ficar em casa...” (E9) } \\
\text { “... acabava por ficar um bocadinho fechada em } \\
\text { mim... me fechar mais em casa, conviver menos... } \\
\text { não me sentir tão confortável em grupo...” (E11) }\end{array}$ & 3 \\
\hline $\begin{array}{l}\text { Cessação } \\
\text { de contrato }\end{array}$ & $\begin{array}{l}\text { “... uma das coisas que pesou, para suspender o } \\
\text { contrato... foi esse fator... não estar numa equipa } \\
\text { na qual eu me sentisse minimamente integrada ou } \\
\text { minimamente bem vinda...” (E11) }\end{array}$ & 1 \\
\hline
\end{tabular}

O impacto das experiências de bullying dos participantes, enquanto vítimas, engloba consequências a nível psicológico, físico e social. A nível psicológico, os participantes relataram ansiedade, stresse, tristeza, irritabilidade, insónias, intenção de mudar de serviço e ponderação; a nível físico, os participantes referiram cansaço e perturbações digestivas. Socialmente, o impacto descrito refere-se ao isolamento e à cessação de contrato.

Estas consequências relatadas pelos participantes têm sido descritas em alguns estudos.
O stresse é referido por vários autores como consequência deste tipo de violência. Hauge, Skogstad e Einarsen (2010) verificaram que o bullying é um potente fator de stresse e que contribui para estados de depressão e ansiedade.

Insónias, ansiedade e irritabilidade foram algumas das queixas referidas na investigação de Carvalho (2007). Um estudo longitudinal, com enfermeiros noruegueses, indicou que a exposição a comportamentos de bullying no início do estudo predizia um aumento dos sintomas posteriores de ansiedade e cansaço (Reknes et. al, 2014).

As perturbações psicossomáticas são descritas como consequência do assédio moral por Hirigoyen (2002), entre as quais as perturbações digestivas.

A autora também refere que o cansaço e perturbações do sono são alguns dos sintomas numa fase inicial do assédio moral.

Quanto à intenção de mudar de serviço, na investigação de Hauge et al. (2010), também se constatou a intenção de turnover. Simons e Mawn (2010) descrevem relatos de enfermeiros sobre abandonar o local de trabalho e até mesmo sobre a ponderação em mudar de profissão. Hirigoyen (2002) descreve o isolamento como um procedimento hostil. No entanto, neste estudo os participantes referem-se ao isolamento como resultante da vivência dos atos de bullying. É descrita a falta de motivação para sair de casa e mesmo para conviver com outras pessoas.

Hirigoyen (2002) refere ainda que pessoas isoladas têm uma maior tendência para serem vítimas de assédio moral. Deste modo, pode-se pensar que o isolamento, enquanto consequência, poderá ser também um fator de risco para o perpetuar da situação nestas pessoas, pela maior vulnerabilidade.

Quanto ao afastamento do local de trabalho, Araújo (2009) constatou que o bullying não é um preditor significativo relativamente à rotatividade. Por outro lado, Hirigoyen (2002) refere que o afastamento, ocasionado por despedimento, negociação, demissão ou pré-reforma, é uma consequência social e económica de assédio moral. Também Hoel, Sheehan, Cooper e Einarsen (2011) referem o turnover como uma consequência de bullying a nível organizacional. 


\section{CONCLUSÃO}

Com este estudo descrevemos as perceções dos enfermeiros sobre bullying no trabalho e o impacto das experiências de bullying na saúde mental e sua vida pessoal. O recurso ao método qualitativo de investigação permitiu compreender o significado deste fenómeno para os enfermeiros, bem como descrever o impacto das vivências relacionadas.

Relativamente ao conceito de bullying no trabalho, a maioria dos participantes enquadra este fenómeno no âmbito da violência psicológica, referindo os colegas de trabalho como principais agressores. Na informação obtida, a frequência e a duração dos comportamentos agressivos não são referidas pelos participantes. As definições de bullying no local de trabalho, encontradas na pesquisa bibliográfica, remetem para várias dimensões, em que a frequência e a duração dos comportamentos agressivos são tidas como fundamentais para distinguir este tipo de violência de todos os outros.

A nível psicológico, os participantes relataram ansiedade, stresse, tristeza, irritabilidade, insónias, intenção de mudar de serviço e ponderação no afastamento do local de trabalho. Cansaço e perturbações digestivas foram as consequências a nível físico. $\mathrm{O}$ isolamento e a cessação de contrato foram as consequências descritas a nível social. A maioria dos participantes referiu não ter tido formação escolar sobre este tema.

\section{IMPLICAÇÕES PARA A PRÁTICA CLÍNICA}

Fica evidenciado de que o bullying é um fator de stresse com as suas implicações nefastas na saúde mental e vida dos enfermeiros e por consequência o desempenho profissional é comprometido, com implicações na qualidade de cuidados em saúde aos indivíduos, famílias e comunidade.

Por isso, consideramos fundamental a capacitação dos enfermeiros para a gestão do stresse e a formação sobre gestão de conflitos, por parte das organizações no âmbito da formação contínua.

No domínio da formação graduada e pós-graduada em Enfermagem evidenciamos a necessidade de formação dos enfermeiros sobre bullying no trabalho, no sentido de consciencializar e sensibilizar alunos e profissionais para esta problemática nos contextos da prática clínica. Sugerimos a continuidade de estudos sobre bullying no trabalho com enfermeiros, através de estudos longitudinais e combinação do método qualitativo e quantitativo a amostras mais numerosas e em diferentes contextos de trabalho.
É essencial o estudo dos efeitos do bullying na saúde mental e vida pessoal dos enfermeiros, bem como o seu impacto na prestação de cuidados. Consideramos, ainda, necessários estudos que visem identificar os ambientes favoráveis ao bullying, e a definição de perfis de agressor e vítima.

\section{REFERÊNCIAS BIBLIOGRÁFICAS}

Araújo, M. S. (2009). Preditores individuais e organizacionais de bullying no local de trabalho (Tese de Doutoramento). Universidade do Minho. Acedido em http://hdl.handle.net/1822/11041

Bardin, L. (2009). Análise de conteúdo (5ª ed.). Lisboa: Edições 70.

Borges, E. M., \& Ferreira, T. J. R. (2015). Bullying no trabalho: Adaptação do Negative Acts QuestionnaireRevised (NAQ-R) em enfermeiros. Revista Portuguesa de Enfermagem de Saúde Mental (13), 25-33.

Carvalho, G. D. (2007). Mobbing: Assédio moral em contexto de enfermagem: Estudo preliminar. Revista Investigação em Enfermagem (15), 43-55. Acedido em http://hdl.handle.net/10198/5331

Einarsen, S., Hoel, H., Zapf, D., \& Cooper, C. L. (2011). The concept of bullying and harassment at work: The European tradition. In S. Einarsen, H. Hoel, D. Zapf, \& C. L. Cooper (Eds.), Bullying and harassment in the workplace: Developments in theory, research, and practice (pp. 3-39). Boca Raton, FL: CRC Press.

European Agency for Safety and Health at Work (2007). Expert forecast on emerging psychosocial risks related to occupational safety and health. Luxemburgo. Acedido em https://osha.europa.eu/en/tools-and-publications/publications/reports/7807118

Hauge, L., Skogstad, A., \& Einarsen, S. (2010). The relative impact of workplace bullying as a social stressor at work. Scandinavian Journal of Psychology, 51, 426-433. doi: 10.1111/j.1467-9450.2010.00813.x

Hirigoyen, M. F. (2002). O assédio no trabalho: Como distinguir a verdade. Cascais: Editora Pergaminho. 
Hoel, H., Sheehan, M. J., Cooper, C. L., \& Einarsen, S. (2011). Organisational effects of workplace bullying. In S. Einarsen, H. Hoel, D. Zapf, \& C. L. Cooper (Eds.), Bullying and harassment in the workplace: Developments in theory, research, and practice (pp. 129-147). Boca Raton, FL: CRC Press.

Leymann, H. (1996). The content and development of mobbing at work. European Journal of Work and Organizational Psychology, 5(2), 165-184. Acedido em http://www.organizedmobbing.com/Leymann1996.pdf

Mitchell, A., Ahmed, A., \& Szabo, C. (2014). Workplace violence among nurses, why are we still discussing this? Literature review. Journal of Nursing Education and Practice, 4(4), 147-150. doi: 10.5430/jnep.v4n4p147

Reknes, I., Pallesen, S., Mageroy, N., Moen, B. E., Bjorvatn, B., \& Einarsen, S. (2014). Exposure to bullying behaviors as a predictor of mental health problems among Norwegian nurses: results from the prospective SUSSHsurvey. International Journal of Nursing Studies, 51, 479-487. doi: 10.1016/j.ijnurstu.2013.06.017

Sá, L. O. (2008). O assédio moral e o burnout na saúde mental dos enfermeiros (Tese de Doutoramento). Instituto de Ciências Biomédicas Abel Salazar, Porto. Acedido em http://repositorio-aberto.up.pt/handle/10216/7223

Saraiva, D. M., \& Pinto, A. S. (2011). Mobbing em contexto de enfermagem. Revista de Enfermagem Referência, III(5), 83-93.
Simons, S. R., \& Mawn, B. (2010). Bullying in the workplace: A qualitative study of newly licensed registered nurses. Journal of the American Association of Occupational Health Nurses, 58(7), 305-311. doi: 10.3928/08910162-20100616-02

Strandmark, M., \& Hallberg, L. (2007). The origin of workplace bullying: Experiences from the perspective of bully victims in the public service sector. Journal of Nursing Management, 15(3), 332-341. doi: 10.1111/j.1365-2834.2007.00662.x

Townsend, T. (2012). Break the bullying cycle. American Nurse Today, 7(1), 12-15.

Zapf, D. (1999). Organisational, work group related and personal causes of mobbing/bullying at work. International Journal of Manpower, 20(1/2), 70-85. doi: $10.1108 / 01437729910268669$

Zapf, D., \& Einarsen, S. (2011a). Individual antecedents of bullying: Victims and perpetrators. In S. Einarsen, H. Hoel, D. Zapf, \& C. L. Cooper (Eds.), Bullying and harassment in the workplace: Developments in theory, research, and practice (pp. 177-200). Boca Raton, FL: CRC Press.

Zapf, D., Escartín, J., Einarsen, S., Hoel, H., \& Vartia, M. (2011b). Empirical findings on prevalence and risk groups of bullying in the workplace. In S. Einarsen, $\mathrm{H}$. Hoel, D. Zapf, \& C. L. Cooper (Eds.), Bullying and harassment in the workplace: Developments in theory, research, and practice (pp. 75-105). Boca Raton, FL: CRC Press.

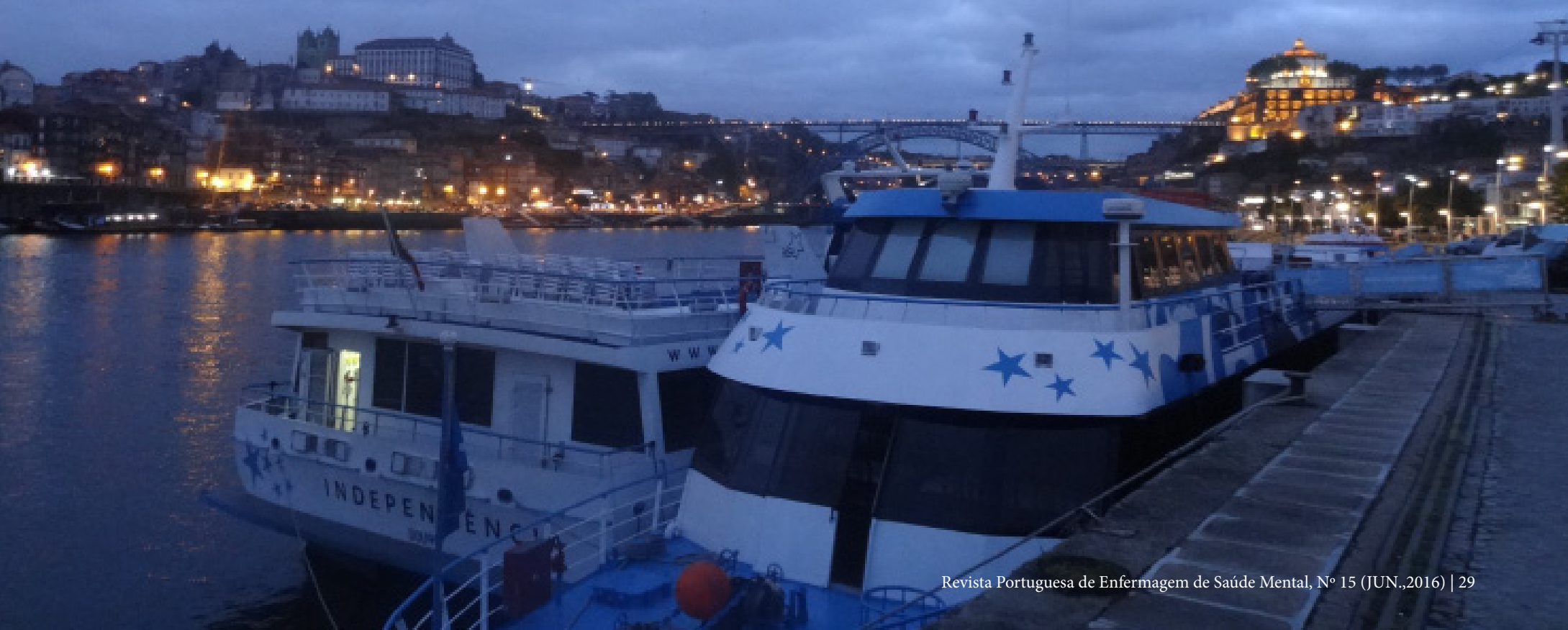

